\title{
FORUM
}

\section{Das irische ,Nein' zum Vertrag von Lissabon: Optionen für die Lösung der neuen Krise}

\author{
Jo Leinen und Jan Kreutz*
}

Das irische ,Nein“ stellt die Europäische Union vor ein großes Problem. Einerseits darf das Votum nicht ignoriert werden, andererseits kann die Zukunft der Zusammenarbeit von 27 Staaten und von 500 Millionen Menschen nicht von 862.415 Personen aus einem Mitgliedstaat blockiert werden. Nach wie vor gilt, dass die Europäische Union mit dem Vertrag von Nizza nicht handlungsfähig genug ist, um die Herausforderungen des 21. Jahrhunderts effizient und effektiv zu lösen. Mit dem Nizza-Vertrag bleiben weiterhin ein Demokratieund ein Transparenzdefizit bestehen, was zunehmend zur Distanz zwischen Bürgern und Entscheidungsträgern in Europa beiträgt. Reformen der Arbeitsweise und der politischen Ausrichtung der Europäischen Union sind deshalb dringend notwendig. Was zur Zeit möglich und konsensfähig ist, wurde mit dem Vertrag von Lissabon beschlossen - eine Neuverhandlung würde nicht zu anderen oder gar besseren Ergebnissen führen. In den nächsten Monaten wird die Debatte über Optionen zur Lösung der Krise eine wichtige Rolle spielen. Nach Verkündung des negativen Ergebnisses wurden Vorschläge in den Raum gestellt, die sich bei näherer Betrachtung als nicht durchführbar oder nicht zielführend erweisen. Vieles spricht für ein zweites Referendum, mit gewissen Zusicherungen und Garantien für Irland. Grundlage der Debatte muss jedoch ein besseres Verständnis der Gründe für die Ablehnung des Vertrages in Irland sein. Um Konsequenzen aus dem ,Nein` zu ziehen, müssen Maßnahmen im Bereich der Kommunikationspolitik getroffen werden und die Entscheidungen der Europäischen Union müssen besser an die Wünsche der Menschen angepasst werden. Darüber hinaus stellt sich angesichts des bisherigen Verlaufs der Ratifizierung des Vertrages von Lissabon die Frage nach der Ratifizierungsmethode von Vertragsänderungen.

\section{Gründe für das irische, Nein'}

Aufschluss über die Gründe für das ,Nein“ in Irland gibt ein kurz nach der Abstimmung veröffentlichter Eurobarometer. ${ }^{1}$ Darin bestätigt sich eine Gewissheit, die sich auch aus Gesprächen mit irischen Experten und Politikern herauskristallisiert: Der Hauptgrund für das Ergebnis war das weitgehende Versagen der Ja-Kampagne. Aufgrund einer ungewöhnlichen Rechtsprechung des höchsten Gerichts im Land darf die Regierung nicht für die Zustimmung in einem Referendum werben. Während die Nein-Kampagne sehr gut organisiert und finanziert war, kam das Ja-Lager mal wieder zu spät und agierte halbherzig. Die politischen Parteien und nationalen Politiker beteiligten sich nur sehr widerwillig an der Debatte. Darüber hinaus spielten wie bei jedem Referendum die Medien eine wichtige Rolle, von denen einige die unreflektierte anti-europäische Propaganda aus Großbritannien übernahmen.

\footnotetext{
Jo Leinen, MdEP, Vorsitzender des Verfassungsausschusses im Europäischen Parlament. Jan Kreutz, Wissenschaftlicher Assistent von Jo Leinen.

1 Vgl. Wolfgang Wessels: Die Debatte nach ,Irland‘: Festhalten an Lissabon, Aufbruch zu Alternativen oder doch Leben mit Nizza?, in diesem Band, S. 312-318.
} 
Diese Konstellation führte dazu, dass die Vertragsgegner simple anti-europäische Parolen vorbrachten, die von den Befürwortern aber nicht engagiert genug entkräftet wurden. Viele der Argumente des Nein-Lagers entbehrten jeglichen Wahrheitsgehaltes. Es wurde behauptet, der Reformvertrag erlaube Abtreibung und Euthanasie. Eine solche Behauptung lässt sich aber weder durch eine besonders weite Auslegung der Grundrechte-Charta noch durch den Rest des Vertrages belegen. Obwohl im Vertrag explizit festgehalten wird, dass jeder Mitgliedstaat selber über die Teilnahme an internationalen Militärmissionen entscheidet und die Verteidigungspolitik sowie die Außenpolitik der Europäischen Union einstimmig beschlossen werden, wurde propagiert, der Vertrag von Lissabon gefährde die Neutralität Irlands. Trotz der weiterhin vorgeschriebenen Einstimmigkeit in Steuerfragen, wurden Ängste geschürt, die Europäische Union könne Irland zwingen, seine niedrigen Steuersätze zu erhöhen. All diese Aspekte haben laut Eurobarometer eine Rolle bei der Ablehnung des neuen Vertrages gespielt.

Einen großen Einfluss hatte auch das Argument des schwindenden Einflusses von Irland in der Europäischen Union. Argumentiert wurde, dass für Irland die Einführung der doppelten Mehrheit ein Verlust sei. Unberücksichtigt blieb dabei, dass die neuen Abstimmungsregeln zu mehr Handlungsfähigkeit der Europäischen Union führen und auch aus irischer Sicht schnellere und bessere Entscheidungen bringen können. Auch der Verlust eines Sitzes im Europäischen Parlament und die Rotation in der Europäischen Kommission wurden thematisiert. Vergessen wurde dabei, dass Irland bereits mit dem Vertrag von Nizza einen Abgeordneten weniger nach Brüssel schicken kann. Nach jetziger Vertragslage wird die Rotation in der Kommission bereits 2009 eingeführt, nicht erst 2014, wie im Vertrag von Lissabon vorgesehen. Nichtsdestotrotz müssen die Sorgen um schwindenden Einfluss - die auch in vielen anderen Mitgliedstaaten bestehen - ernst genommen werden. Ein Ausweg wäre hier ein Beschluss des Europäischen Rates über die künftige Rotationsregel und die Struktur der Kommission. So wäre eine Struktur mit Kommissaren und Stellvertretenden Kommissaren ohne Stimmrecht (ähnlich wie Minister und Staatssekretär in Deutschland) denkbar. Damit hätte jeder Mitgliedstaat eine politische Persönlichkeit in der Kommission, ohne die Handlungsfähigkeit des konsensorientierten Kommissionskollegs zu schwächen.

Es besteht ein scheinbarer Widerspruch zwischen der hohen Zustimmung der Iren zur Europäischen Union im Allgemeinen und der Ablehnung des neuen Vertrages. Dies lässt sich dadurch erklären, dass die wirtschaftlichen Vorteile der Europäischen Union für die Iren spürbar sind. Wohl folgten viele Iren der Logik: „Wenn die Europäische Union schon gut funktioniert, warum sollte man das Risiko eingehen, irgendetwas zu ändern.“ Beklagt wurde von Politikern, die für den Vertrag auf die Straße gingen, die erschreckende Unkenntnis über die Europäische Union und ihre Arbeitsweise. Die Notwendigkeit von institutionellen und strukturellen Reformen konnte nicht erklärt werden, weil Grundkenntnisse über den Status quo nicht vorhanden waren. Die Ursache ist darin zu suchen, dass die Europäische Union keine prominente Rolle in den Medien spielt und auch von den nationalen Politikern kaum erwähnt wird. Dies gilt übrigens nicht nur für Irland.

Eine nicht zu unterschätzende Rolle für das ,Nein' in Irland lag aber auch in der allgemeinen Stimmung gegenüber der eigenen Regierung. Es gibt ein komplexes Geflecht aus Misstrauen gegenüber der politischen Klasse, Unzufriedenheit mit der Unausgewogenheit der europäischen Politiken sowie wirtschaftlichen und sozialen Existenzängsten. Angesichts der fortschreitenden Globalisierung sinkt die Handlungsfähigkeit nationaler Regierungen. Anstatt in der Europäischen Union die Lösung für diese Herausforderung zu erkennen, wird sie als Teil des Problems dargestellt. Von einigen Vertragsgegnern wurde die soziale Komponente thematisiert, die für die Ablehnung des Verfassungsvertrages in Frankreich eine 
wichtige Rolle gespielt hat. Der jahrelange wirtschaftliche Boom in Irland ist an sein Ende gelangt. Steigende Arbeitslosigkeit und hohe Preise - insbesondere für Energie und Nahrungsmittel - machten in den letzten Monaten den erfolgsverwöhnten Iren zu schaffen. Die Sorge, die Politik der Europäischen Union habe negative Auswirkungen auf die soziale Situation der Bürger, ist in Irland verbreitet. Insbesondere die kürzlich getroffenen Entscheidungen des Europäischen Gerichtshofs haben für viel Aufregung gesorgt und einige der Einzelgewerkschaften veranlasst, gegen den Vertrag zu mobilisieren. Dabei würde die Europäische Union gerade durch den Vertrag von Lissabon einen größeren Beitrag zu sozialem Fortschritt leisten können.

\section{Die Lösungsoptionen}

Am Freitag den 13. Juni 2008, dem Tag als die enttäuschenden Abstimmungsergebnisse langsam aber sicher Gewissheit wurden, stellte sich erneut die Frage nach dem Plan B. Angesichts des negativen Ergebnisses wurden Vorschläge gemacht, die als nicht durchführbar oder politisch erstrebenswert verworfen werden müssen. Dazu gehört beispielsweise die Idee, Irland aus der Union auszuschließen. Zwar gäbe es juristisch die Möglichkeit, dass 26 Mitgliedstaaten aus der Europäischen Union austreten und auf Grundlage des Vertrages von Lissabon eine neue ,Europäische Union` gründen. Aber der Zusammenarbeit in der Europäischen Union würde es schaden, auf eine demokratische Abstimmung mit solch einer Operation zu reagieren und somit eines der pro-europäischsten Völker aus der Europäischen Union zu drängen. Darüber hinaus würden viele Mitgliedstaaten einen solchen Schritt nicht akzeptieren. Auch Forderungen, den Vertrag von Lissabon zu beerdigen oder neu zu verhandeln, müssen zurückgewiesen werden. Die Europäische Union benötigt die im Lissabonner Vertrag enthaltenen Reformen. Neue Verhandlungen würden angesichts der politischen Voraussetzungen nicht zu besseren Ergebnissen führen.

Eine prominente Forderung ist die nach einem Kerneuropa oder einem Europa der zwei Geschwindigkeiten. Sie wurde in den letzten fünfzehn Jahren immer dann erhoben, wenn eine Lösung einer EU-Krise nicht in Sicht war. Diese Option bringt uns aber bei der Überwindung des jetzigen Problems nicht weiter. Das Ratifizierungsverfahren für den Vertrag von Lissabon ist in den meisten Mitgliedstaaten abgeschlossen, auch im traditionell europaskeptischen Großbritannien. Es wäre nicht verantwortlich, zu solch einem Zeitpunkt die Idee aufzugeben, die gesamte Europäische Union auf eine neue Integrationsstufe zu bringen. Allerdings wird es auch in Zukunft neue Möglichkeiten für eine differenzierte Integration geben, wie in der Vergangenheit mit dem ,Euro' und dem ,Schengenraum‘. Mit dem neuen Vertrag werden die Regeln für eine verstärkte Zusammenarbeit vereinfacht und auf neue Bereiche ausgedehnt, beispielsweise auf die Gemeinsame Sicherheits- und Verteidigungspolitik. Ein Europa der zwei Geschwindigkeiten ist keine Voraussetzung für das Inkrafttreten des Vertrages von Lissabon, sondern seine Ratifizierung eine Voraussetzung für das bessere Funktionieren einer differenzierten Integration.

Mit einer der vorgebrachten Optionen kann der Spagat zwischen Respekt der in Irland vorgebrachten Meinungen und Gewährleistung der Handlungsfähigkeit der Europäischen Union gelingen: Die Regierung in Dublin muss das Referendum gründlich analysieren und den Partnern einen Vorschlag zur Überwindung der Blockade machen. Auf die mit dem ,Nein' zum Ausdruck gebrachten Sorgen der Iren kann eingegangen werden, entweder durch eine politische Erklärung oder notfalls auch durch ein Protokoll, das dem Vertrag angehängt wird. In einer politischen Erklärung könnten gewisse Inhalte des neuen Vertrages noch einmal unterstrichen werden, beispielsweise die Nichteinmischung der Europäischen 
Union in Fragen wie Abtreibung und Euthanasie, die Einstimmigkeit in der Steuerpolitik oder die Festschreibung der irischen Neutralität. Die Befürchtungen vor Sozialdumping könnten in einer Erklärung über ,Sozialen Fortschritt in der EU` aufgegriffen werden. Darüber hinaus kann es eine Klarstellung zur Vertretung aller Mitgliedstaaten in der Europäischen Kommission geben, ohne die Idee der Rotation aufzugeben. Sollte ein ,Paket‘ mit Erklärungen nicht ausreichend sein, könnte in einem Protokoll festgehalten werden, dass Irland aus einer gemeinsamen Politik aussteigt oder nur unter bestimmten Bedingungen teilnimmt.

Aufgrund der irischen Verfassung werden die Bürgerinnen und Bürger von Irland wohl ein zweites Mal an die Urnen gerufen werden müssen. Auswirkungen auf den Erfolg eines zweiten Referendums kann die Frage haben, die von der Regierung gestellt wird. Die ehrlichste und deutlichste Fragestellung wäre, ob Irland in der neuen Europäischen Union mit dem Vertrag von Lissabon mitmachen oder ob es zurückbleiben will. Die Gefahr bei solch einer Formulierung ist sicherlich, dass etliche Bürger aus Trotz gegen die Europäische Union stimmen würden. Denkbar ist auch, dass die Regierung die Errungenschaften und Neuerungen des Vertrages zusammenfasst und fragt, ob die Bürger diese befürworten oder ablehnen. Wichtig ist allemal eine ehrliche Auseinandersetzung über die Vor- und Nachteile des neuen Vertrages im Vergleich zum Vertrag von Nizza und über die Konsequenzen eines zweiten ,Neins'. Ein erheblich größerer Einsatz der Regierung, der nationalen Politiker und der Zivilgesellschaft für ein ,Ja' ist jedenfalls notwendig, um den Erfolg bei einer zweiten Abstimmung zu erreichen.

\section{Aus den drei ,Neins' müssen Konsequenzen gezogen werden}

Die negativen Referenden in Frankreich, den Niederlanden und jetzt in Irland und die dadurch ausgelösten Krisen können nicht ignoriert werden. Die nationalen Regierungen und die EU-Institutionen müssen Konsequenzen ziehen. Einerseits wird immer deutlicher, dass wir mit der jetzigen Ratifizierungsmethode nicht weiterkommen. ${ }^{2}$ Andererseits ist sehr offensichtlich, dass wir ein großes Kommunikationsproblem haben. Nationale Regierungen und die politische Klasse auf allen Ebenen betreiben nach wie vor ,EU-Bashing ' und tragen nicht dazu bei, die Bürger über europäische Politik seriös zu informieren. Bei der Bitte an die Bürger um Unterstützung für ein neues europäisches Projekt wundern sie sich, wenn diese mit ,Nein" stimmen. Auch in vielen Medien taucht die Europäische Union nur als Ort für Skandale und zur Verabschiedung von unliebsamen Regulierungen auf. Selbst zivilgesellschaftliche Vertreter sind oft nur halbherzig bereit, für die Idee eines vereinten Europas zu werben.

Angesichts der Tatsache, dass sich eine große Mehrheit der Unionsbürger schlecht informiert fühlt, ist es verantwortungslos, dass sich einige Mitgliedstaaten nach wie vor gegen eine gemeinsame Kommunikationspolitik sperren. Seit Jahren werden entsprechende Vorschläge der Europäischen Kommission und des Europäischen Parlaments zurückgewiesen. Die Argumentation, Kommunikation über Europa sei ausschließlich eine nationale Angelegenheit, entspricht dem Denken von vor 50 Jahren. Wir leben in einer Mehrebenendemokratie und jede Ebene muss die Möglichkeit haben, sich den Bürgern mitzuteilen und für die getroffenen Entscheidungen zu werben. Darüber hinaus ist es bedenklich, dass kein einheitlicheres Auftreten des Europäischen Parlaments, der Europäischen Kommission und des Ministerrats in der Öffentlichkeit existiert. Die Kommunikation über getroffene Ent-

2 Dies wird im Beitrag zur partizipativen Demokratie von Jo Leinen und Jan Kreutz in diesem Band weiter ausgeführt, siehe Seite 241-253. 
scheidungen wird von den Regierungen als Faustpfand der eigenen Macht reserviert. Spätestens seit dem Ergebnis in Irland muss eine echte europäische Informationspolitik vorangetrieben werden.

Um mit den Worten Roman Herzogs zu sprechen: ,Es muss ein Ruck durch Europa gehen!' Wir brauchen mehr Engagement von Politikern und Meinungsführern auf lokaler, Landes-, Bundes- und europäischer Ebene. Die Vision eines friedlich geeinten europäischen Kontinents, auf dem alle Bürger in Sicherheit leben und einen angemessenen Lebensstandard haben, bleibt bestehen. Sie muss nur immer wieder neu in das Bewusstsein von Bürgern und Entscheidungsträgern gebracht werden. Die letzten Jahre wurde viel Energie und Zeit für die Lösung der institutionellen und strukturellen Probleme aufgewandt. Dabei wurde wohl zu oft das große Ganze vergessen. Darauf müssen wir uns in der nächsten Zeit zurückbesinnen, sonst werden wir bei den europäischen Wahlen eine herbe Überraschung erleben, sowohl in einer noch geringeren Wahlbeteiligung als auch in einem noch größeren Erfolg für die Europaskeptiker und Populisten in verschiedenen Mitgliedstaaten. 30 Jahre nach der Einführung der ersten Direktwahl zum Europäischen Parlament werden die nächsten Europawahlen im Juni 2009 ein echter Test für die Stabilität und Zukunftsfähigkeit des europäischen Projekts.

\section{Europäischer Einigungsprozess}

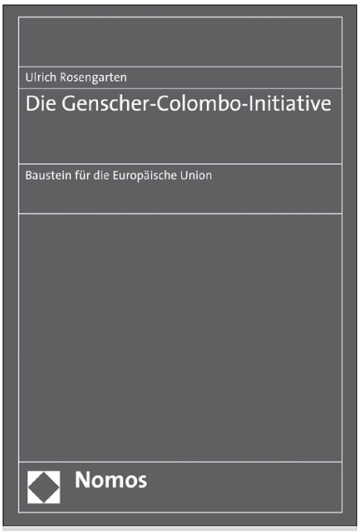

\section{Die Genscher-Colombo-Initiative}

Baustein für die Europäische Union

Von Ulrich Rosengarten

2008, 219 S., brosch., 39,- €, ISBN 978-3-8329-3364-7

Europa ist kein Kinderspiel! Daher ist es nützlich zu erfahren, wie kompliziert es ist, Europa voranzubringen. Der politische Anstoß, den Genscher und Colombo 1981 mit ihrer Europainitiative dem europäischen Einigungsprozess gaben, musste erhebliche Widerstände überwinden mit denen die Impulsgeber von Seiten selbst befreundeter europäischer Partner konfrontiert wurden.

Bitte bestellen Sie bei Ihrer Buchhandlung oder bei Nomos 